\title{
Turning a challenge into an opportunity: pulmonary rehabilitation and socioeconomic deprivation
}

\author{
Kylie N Johnston, ${ }^{1}$ Marie T Williams ${ }^{2}$
}

The efficacy of pulmonary rehabilitation has been established for people with COPD such that the addition of further controlled clinical trials comparing pulmonary rehabilitation with usual care is considered unnecessary. ${ }^{1}{ }^{2}$ How well the degree of health benefit reported within clinical trials of pulmonary rehabilitation translates into real-life settings is more difficult to ascertain. In real-world clinical environments, the effectiveness of pulmonary rehabilitation for people with COPD is likely to be confounded by heterogeneity in national healthcare systems, structure and staffing of rehabilitation programmes and participant characteristics of those referred to, accessing and completing pulmonary rehabilitation. While pragmatic clinical trials might provide local intelligence on pulmonary rehabilitation effectiveness, census-level surveys of clinical outcomes and sociodemographic data or pulmonary rehabilitation registries, such as those established in the USA ${ }^{3}$ and proposed in Australia ${ }^{4}$ permit associations between service provision, demographic characteristics and clinical outcomes to be explored at the population level.

The value of such population-level approaches is evident in the paper by Steiner and colleagues ${ }^{5}$ published in Thorax. Using data from the 2015 National COPD Clinical Audit Programme (England and Wales), Steiner and colleagues present an analysis of uptake and performance of participants across 230 pulmonary rehabilitation services, ${ }^{6}$ including over 7000 participants with COPD. ${ }^{7}$ Steiner and colleagues have reported that completion (defined as attending a discharge assessment) of pulmonary rehabilitation is lower in those with greater levels of socioeconomic deprivation. Where programmes are not 'completed', the optimal benefits of rehabilitation in terms of quality of life,

${ }^{1}$ Sansom Institute for Health Research, University of South Australia, Adelaide, Australia; ${ }^{2}$ Alliance for Research in Exercise, Nutrition and Activity (ARENA), Sansom Institute for Health Research, University of South Australia, Adelaide, Australia

Correspondence to Dr Kylie Johnston, School of Health Sciences, Sansom Institute for Health Research, University of South Australia, GPO Box 2471, Adelaide, SA 5001, Australia; kylie.johnston@unisa.edu.au breathlessness and exercise capacity are not expected to be gained. This is consistent with the results shown in other populationbased studies where people with COPD and lower socioeconomic status attain poorer outcomes across the board, including greater mortality, more hospitalisations, less use of inhaled medication and poorer health-related quality of life. ${ }^{8}$ It is worth noting that within trials of pulmonary rehabilitation, there is currently no consistency in a priori criteria for 'sufficient' session attendance (50\%-100\% reported), and while increased attendance rates might be expected to result in greater attainment of pulmonary rehabilitation benefits, to date there is no clear evidence of doseresponse. $^{9}$

Low referral to and uptake of pulmonary rehabilitation are the recognised issues in general and result in $<5 \%$ of people with COPD receiving this intervention. ${ }^{10}$ For the subgroup of socioeconomically deprived people with COPD, referral and uptake rates may be even lower. In the discussion of their findings, Steiner and colleagues compare the deprivation profile of those who attended a baseline appointment for pulmonary rehabilitation with the profile of those accessing hospital services for COPD (data also part of the National COPD Audit ${ }^{11}$ ). The group admitted to hospital had a more socioeconomically deprived profile, suggesting that the most socioeconomically disadvantaged people with COPD were under-represented in attendance at a first appointment at pulmonary rehabilitation. Barriers to first attendance at pulmonary rehabilitation associated with socioeconomic status may arise at multiple points. Referral to pulmonary rehabilitation may not be offered by primary care physicians, ${ }^{12}$ and the inclusion of referral as part of a defined post-exacerbation pathway is known to be low (2\% of referrals to pulmonary rehabilitation in England and Wales; $0.62 \%$ in the USA $\left.{ }^{10}\right)$. In the England and Wales audit data, only $69 \%$ of those referred went on to attend a first appointment at pulmonary rehabilitation, citing lack of perceived benefit, clinically inappropriate referral and comorbid medical conditions as predominant reasons. ${ }^{7}$ Of who attended the first appointment, a further $40 \%$ did not complete the programme, again due to lack of perceived benefit, comorbidities and COPD exacerbations. ${ }^{7}$

However, while people with COPD with socioeconomically deprived profiles were less likely to attend a discharge assessment, Steiner and colleagues report that the gains made in health status and functional exercise capacity on completion of pulmonary rehabilitation did not differ by socioeconomic strata (after accounting for other associated baseline variables). There is value in examining the degree of clinical improvement gained from pulmonary rehabilitation in this real-world sample, where programme eligibility criteria may be less restrictive than in randomised controlled trials of pulmonary rehabilitation. The eligibility criteria of the trials that contributed to the definitive review of pulmonary rehabilitation effectiveness ${ }^{1}$ have not themselves been systematically examined. McCarthy et $a l^{1}$ reported a male gender bias $(69 \%$ of all participants in included studies), and data extraction tables indicate many studies excluded those with locomotor problems, musculoskeletal or orthopaedic disorders, lack of motivation or any medical conditions that might limit exercise tolerance. In contrast, people with multimorbidity, locomotor problems and mental health disorders are present in these national audit data, and while comorbidities were unilaterally associated with lower completion rates, their presence did not have an overall negative association with clinical outcomes.

As $94 \%$ of the audit population selfidentified as white British, ${ }^{7}$ a further gap highlighted by this paper is the need for implementation of pulmonary rehabilitation and outcome data for ethnically diverse and indigenous populations. Non-white British people were underrepresented among pulmonary rehabilitation participants in England and Wales compared with the general population. ${ }^{13}$ Similarly, indigenous people are nearly absent from pulmonary rehabilitation programmes and associated research, ${ }^{14}$ yet have health disparity beyond what is associated with socioeconomic status alone. Barriers to implementation of pulmonary rehabilitation here include staffing, resourcing and sustainability of programmes in rural and remote settings ${ }^{15}$ along with addressing cultural meaning and relevance for indigenous participants. ${ }^{14} 15$

The challenge from this study as articulated by the authors is to increase referral and provision of pulmonary rehabilitation services, with priority given to those in circumstances of socioeconomic deprivation. 
Given that COPD is disproportionally represented in lower socioeconomic groups, worldwide ${ }^{8}$ examination of the influence of social factors on implementation of pulmonary rehabilitation has been lacking to date. Recommendations of Steiner and colleagues include 'raising awareness of the benefits of pulmonary rehabilitation to patients from deprived areas and changing referral practice among healthcare workers who provide care in these areas'. 5 The latter may be facilitated by clear processes including a referral pathway from hospital care and financially incentivised support for making pulmonary rehabilitation a usual part of primary care for people with COPD in these areas. ${ }^{12}$

Removing the barriers and promoting the benefits of pulmonary rehabilitation to people with COPD in areas of socioeconomic deprivation will involve addressing known general external barriers such as transport and illness. ${ }^{16}$ However, strategies specific to this target population need to recognise the socioeconomic gradient in adoption of health-promoting behaviours including diet, physical activity and smoking cessation. Behaviour change interventions directed toward physical activity in lower socioeconomic groups show an overall small positive effect, ${ }^{17}$ but lower than when such interventions are applied in the general population. Targeting specific behaviour change strategies that have demonstrated effectiveness in lower socioeconomic populations may be a way forward. As a simple example, a qualitative study in the Netherlands that explored perceptions on health behaviours of people with low socioeconomic status found motivation to change was more induced by health complaints than for preventive purposes; being part of a group of their peers was seen as supportive of lifestyle change. ${ }^{18}$ Both of these aspects could be readily highlighted in the conversations between referrers and the people who stand to benefit from pulmonary rehabilitation.

Despite advances in clinical management, underneath the 'lack of perceived benefit' of pulmonary rehabilitation expressed by both patients and practitioners may remain a therapeutic nihilism that has long been associated with both COPD and the symptom of chronic breathlessness. ${ }^{19}$ It is also feasible that this attitude is magnified in environments of socioeconomic deprivation and will require targeted strategies to address it. An additional contributor could be the poor health literacy associated with lower socioeconomic status, which refers to ability to obtain, process and understand basic health-related information and services needed to make appropriate healthcare decisions. $^{20}$ In people with COPD, poor health literacy is associated with worse health outcomes and learned helplessness, ${ }^{20}$ suggesting another possible mechanism for the lack of perceived benefit to be gained from pulmonary rehabilitation.

Steiner and colleagues are among the first, but not likely to be the last, to provide clear evidence of health disparity by socioeconomic deprivation in access and completion of pulmonary rehabilitation. The opportunities that national audits or registries provide are invaluable and raise the real possibility of being able to identify groups and processes that need prioritising. On the foundation of this audit analysis in particular, we need to develop strategies to improve targeting of pulmonary rehabilitation to be meaningful and accessible to the most socially disadvantaged people with COPD.

Contributors KNJ and MTW jointly developed the concepts in this editorial. KNJ wrote the first draft. KNJ and MTW reviewed and modified the draft and agreed on the final version.

Competing interests None declared.

Provenance and peer review Commissioned; externally peer reviewed.

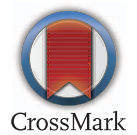

To cite Johnston KN, Williams MT. Thorax 2017;72:493-494.

Published Online First 14 February 2017

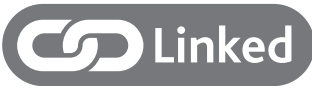

- http://dx.doi.org/10.1136/thoraxjnl-2016-209376

Thorax 2017;72:493-494.

doi:10.1136/thoraxjnl-2016-209798

\section{REFERENCES}

1 McCarthy B, Casey D, Devane D, et al. Pulmonary rehabilitation for chronic obstructive pulmonary disease. Cochrane Database Syst Rev 2015;(2):CD003793.

2 Lacasse Y, Cates CJ, McCarthy B, et al. This Cochrane Review is closed: deciding what constitutes enough research and where next for pulmonary rehabilitation in COPD. Cochrane Database Syst Rev 2015;(11):ED000107.

3 American Association of Cardiovascular and Pulmonary Rehabilitation. Outpatient Pulmonary Rehabilitation Data Registry. 2016. https://www. aacvpr.org/Registry/Pulmonary-Rehabilitation-Registry (accessed Dec 2016).

4 Marks G, Reddel H, Guevara-Rattray E, et al. Australian Institute of Health and Welfare. Monitoring pulmonary rehabilitation and long-term oxygen therapy for people with chronic obstructive pulmonary disease (COPD) in Australia: a discussion paper. Canberra: AlHW, 2013; Cat. no. ACM 29.

5 Steiner MC, Lowe D, Beckford K, et al. Socioeconomic deprivation and the outcomes of pulmonary rehabilitation in England and Wales.
Thorax. 2017;72:530-7.

6 Steiner M, Holzhauer-Barrie J, Lowe D, et al. Pulmonary Rehabilitation: Time to breathe better. National Chronic Obstructive Pulmonary Disease (COPD) Audit Programme: Resources and organisation of Pulmonary Rehabilitation services in England and Wales 2015. National organisational audit report. London: RCP, 2015.

7 Steiner M, Holzhauer-Barrie J, Lowe D, et al. Pulmonary Rehabilitation: Steps to breathe better. National Chronic Obstructive Pulmonary Disease (COPD) Audit Programme: Clinical audit of Pulmonary Rehabilitation services in England and Wales 2015. National clinical audit report. London: RCP, 2016.

8 Pleasants RA, Riley IL, Mannino DM. Defining and targeting health disparities in chronic obstructive pulmonary disease. Int J Chron Obstruct Pulmon Dis 2016;11:2475-96.

9 Williams MT, Lewis L, McKeough Z, et al. Reporting of exercise attendance rates for people with chronic obstructive pulmonary disease: a systematic review. Respirology 2014;19:30-7.

10 Nishi SP, Zhang W, Kuo Y, et al. Pulmonary rehabilitation utilization in older adults with chronic obstructive pulmonary disease, 2003-2012. I Cardiopulm Rehabil Prev 2016;36:375-82.

11 Stone RA, Holzhauer-Barrie J, Lowe D, et al. COPD: Who cares matters. National Chronic Obstructive Pulmonary Disease (COPD) Audit Programme: Clinical audit of COPD exacerbations admitted to acute units in England and Wales 2014. National clinical audit report. London: RCP, 2015.

12 Johnston KN, Young M, Grimmer K, et al. Barriers to, and facilitators for, referral to pulmonary rehabilitation in COPD patients from the perspective of Australian general practitioners: a qualitative study. Prim Care Respir J 2013:22:319-24.

13 Office for National Statistics. Ethnicity and national identity in England and Wales: 2011. 2012. https:/l www.ons.gov.uk/peoplepopulationandcommunity/ culturalidentity/ethnicity/articles/ethnicityandnational identityinenglandandwales/2012-12-11 (accessed Dec 2016).

14 Levack WM, Jones B, Grainger $R$, et al. Whakawhanaungatanga: the importance of culturally meaningful connections to improve uptake of pulmonary rehabilitation by Māori with COPD—a qualitative study. Int I Chron Obstruct Pulmon Dis 2016;11:489-501.

15 Johnston $\mathrm{CL}$, Maxwell LJ, Alison JA. Establishing and delivering pulmonary rehabilitation in rural and remote settings: The opinions, attitudes and concerns of health care professionals. Aust J Rural Health 2016;24:106-14.

16 Keating $A$, Lee AL, Holland AE. Lack of perceived benefit and inadequate transport influence uptake and completion of pulmonary rehabilitation in people with chronic obstructive pulmonary disease: a qualitative study. J Physiother 2011;57:183-90.

17 Bull ER, Dombrowski SU, McCleary N, et al. Are interventions for low-income groups effective in changing eating, physical activity and smoking behaviours? A systematic review and meta-analysis. BMJ Open 2014:4:e006046.

18 Bukman AJ, Teuscher D, Feskens EJ, et al. Perceptions on healthy eating, physical activity and lifestyle advice: opportunities for adapting lifestyle interventions to individuals with low socioeconomic status. BMC Public Health 2014;14:1036.

19 Currow DC, Abernethy AP. Therapeutic nihilism: the next hurdle in improving the treatment of breathlessness. Curr Opin Support Palliat Care 2011:5:69-70.

20 Omachi TA, Sarkar U, Yelin EH, et al. Lower health literacy is associated with poorer health status and outcomes in chronic obstructive pulmonary disease. J Gen Intern Med 2012;28:74-81. 